\title{
Transplantation of Olfactory Ensheathing Cells into Spinal Cord Lesions Restores Breathing and Climbing
}

\author{
Ying Li, ${ }^{1}$ Patrick Decherchi, ${ }^{2}$ and Geoffrey Raisman ${ }^{1}$ \\ ${ }^{1}$ Norman and Sadie Lee Research Centre, Division of Neurobiology, National Institute for Medical Research, Medical Research Council, London NW7 1AA, \\ United Kingdom, and 'Faculté des Sciences du Sport de Marseille-Luminy, Unité Propre de Recherche de l'Enseignement Supérieur EA3285 Déterminants \\ Physiologiques de l'Activité Physique-Institut Federatif de Recherche E. J. Marey, Université de la Méditerranée (Aix-Marseille II), 13288 Marseille cedex \\ 09, France
}

One of the most devastating effects of damage to the upper spinal cord is the loss of the ability to breathe; patients suffering these injuries can be kept alive only with assisted ventilation. No known method for repairing these injuries exists. We report here the return of supraspinal control of breathing and major improvements in climbing after the application of a novel endogenous matrix transfer method. This method permits efficient transfer and retention of cultured adult rat olfactory ensheathing cells when transplanted into large lesions that destroy all tracts on one side at the upper cervical level of the adult rat spinal cord. This demonstrates that transplantation can produce simultaneous repair of two independent spinal functions.

Key words: spinal cord; breathing; olfactory ensheathing cells; repair; adult; transplantation

\section{Introduction}

Spinal cord lesions above the level of the third cervical segment sever the continuity of the bulbospinal respiratory pathways, which carry the descending rhythmic impulses needed to activate the phrenic motoneuron pool lying in the ventral gray matter between the third and fifth cervical segments. Clinically, such patients become dependent on continuously assisted ventilation. A number of recent studies (Li et al., 1997, 1998; Ramón-Cueto et al., 2000; Lu et al., 2002) have put forward evidence that structural and functional repair of the adult rat spinal cord can be achieved by transplantation of glial cells obtained from the primary olfactory pathways, and this has been proposed as a model for clinical application (Raisman, 2001). From a practical point of view, it would be difficult to study the repair of the supraspinal respiratory pathways in animals that need to be maintained on chronic artificial ventilation as a result of complete loss of function. We therefore sought partial lesions that would consistently remove a part of the respiratory pathway while sparing a sufficient supraspinal control mechanism to maintain respiratory function in normal cage behavior. For this purpose we took advantage of bulbospinal respiratory pathways that are bilaterally symmetrical. High spinal hemisection abolishes phrenic nerve and diaphragm function on the side of the lesion but leaves the opposite side functionally intact.

Received Sept. 13, 2002; revised Nov. 4, 2002; accepted Nov. 13, 2002.

This work was supported by the British Neurological Research Trust, the Institut pour la Recherche sur la Moelle Epinière, and the International Spinal Research Trust. We thank Grant Roalfe for cell culture, John Atkinson for the vector, Laiwen Fu for histology work, and Dr. Daqing Li for collaboration.

Correspondence should be addressed to Dr. Geoffrey Raisman, Division of Neurobiology, National Institute for Medical Research, The Ridgeway, Mill Hill, London NW7 1AA, UK. E-mail: graisma@nimr.mrc.ac.uk.

Copyright $\odot 2003$ Society for Neuroscience $\quad$ 0270-6474/03/230727-05\$15.00/0

\section{Endogenous matrix transfer}

Our previous results were obtained with injections of cell suspensions into lesions of the corticospinal tract, where the functional deficits were assessed in a directed forepaw reaching task ( $\mathrm{Li}$ et al., 1997). In the present study we used knife cuts to sever the entire spinal gray and white matter on one side at the level of the second cervical segment in adult rats. The larger amounts of tissue destruction are more comparable with those encountered in clinical practice. In addition to respiration, the larger lesions enable us to study a wider range of functions, such as the coordinated movements used in climbing. From a practical point of view, however, the injection of cell suspensions, which was adequate to repair small, circumscribed electrolytic lesions of the corticospinal tract, would be highly inefficient for the large hemisections, which are, in effect, semicircular cuts, and open along their entire periphery. To permit retention of the transplanted cells in these larger lesions, we have developed a method of transplantation of the primary cultured cells by scraping off the matrix that cells produce in culture, and in which they are embedded. Using the matrix of the cell (i.e., endogenous matrix) for transfer has several advantages. It preserves the two cell types (see below) in the proportions they develop in culture, it avoids the requirement for adding extraneous material, and additionally, from a practical point of view, it not only avoids loss of cells during transplantation but also prevents diffusion of the cells away from the transplant site after operation.

\section{Note on terminology}

The present consensus of terminology refers to the p75-positive, spindle-shaped ("Schwann-like") cells of the olfactory nerves as olfactory ensheathing cells (OECs). A quite distinct cell type that is fibronectin-positive, and with all of the characteristics of a fibroblast, is present (1) in intimate relationship with the OECs in 
the olfactory nerves in situ, (2) in the cell cultures derived from them, and (3) in our ultrastructural analysis of transplants in the corticospinal tract (Li et al., 1998). We believe that the presence of both cell types is necessary for the transplants to express their reparative properties. In our previous publication we called these two cell types $\mathrm{S}$ cells and A cells, respectively. We propose that a more explicit identification is to refer to the second cell type as olfactory nerve fibroblasts (ONFs).

\section{Materials and Methods}

Operation. Eighty-two adult female, inbred AS strain rats were used. The supraspinal muscles were parted in the midline, the atlanto-occipital membrane was incised, and one-half of the upper cervical spinal cord was transected with the use of an operating microscope and a small knife (model 10072-12; Fine Science Tools GmbH, Heidelberg, Germany), taking care to avoid damage to the dorsal median blood vessel. This operation has the advantage that there is virtually no bleeding and no damage to bone or ligament. It can be repaired by skin suture and causes minimal if any local operative discomfort.

Protocol for cell transfer. In 40 animals the lesions were filled at the time of operation with a mixture of $\sim 500,000$ OECs and ONFs in equal number that were derived from tissue dissected from the outer nerve and glomerular layers of the rat olfactory bulb. The cells were trypsinized ( $1 \%$ trypsin for $30 \mathrm{~min}$ at $37^{\circ} \mathrm{C}$ ), plated onto $35 \mathrm{~mm}$ dishes coated with polyL-lysine, and cultured for $14-17 \mathrm{~d}$ as described by Li et al. (1998). The initial plating density is critical for the cells to produce a sufficiently firm matrix to allow transfer into the lesion site. The pooled tissue from six bulbs yields approximately four dishes. The optimal cell mixture is obtained between 14 and $17 \mathrm{~d}$ culture in DMEM-F12 medium with $10 \%$ fetal calf serum (3133-028; Invitrogen, San Diego, CA). At that time, each dish yields $\sim 1.5$ million cells, of which $\sim 50 \%$ are p 75 -positive OECs and $50 \%$ are fibronectin-positive ONFs (Li et al., 1998). They were embedded in a matrix of their own production and covered the dish to a thickness of $\sim 20 \mu \mathrm{m}$. This cell-containing matrix is scraped off the dish with a polythene scraper (3010; Costar, Corning, NY), gathered into a mass of $\sim 5$ $\mathrm{mm}$, and cut into four to five pieces. Using watchmaker's forceps, the pieces are lifted out of the dish and transferred directly into the lesion area. Each piece is able to fill a hemisection lesion of $\sim 4 \mathrm{~mm}^{2}$, with an edge separation of $\sim 2 \mathrm{~mm}$.

Compared with our previous experiments with injection of a suspension of cells into corticospinal tract lesions (Li et al., 1997), the present matrix transfer protocol minimizes loss of cells remaining in the container and the micropipette during transfer. In the previous experiments in which a cell suspension was injected into corticospinal tract lesions with a volume of $\sim 1 \mathrm{~mm}^{3}$, one dish of cells was needed for each transplant. In the present hemisections, with a lesion volume of $\sim 8 \mathrm{~mm}^{3}$, one dish of cells was sufficient for up to five transplants (a 40-fold increase in efficiency). However, the overall advantage over using a cell suspension is effectively much greater, because the matrix retains the cells in the lesion site, thereby preventing postoperative loss by diffusion and obviating the need for introducing any exogenous matrix materials. One day before transplantation, the cells were transfected with adenovirus harboring enhanced green fluorescent protein (eGFP) (available from Clontech, Palo Alto, CA). This labels $\sim 50 \%$ of the cells and provides a strong fluorescent label for up to 12-14 d after transplantation (Ruitenberg et al., 2002).

Electrophysiological recording of respiratory rhythm in the phrenic nerve. After 2 months of survival, the rats were deeply anesthetized, blood pressure was monitored continuously, the rats were endotracheally intubated, and the phrenic nerve on the operated side was exposed, immersed in a pool of warm paraffin oil, cut distally, and placed on bipolar tungsten electrodes (Decherchi et al., 1996). The compound action potential of $\sim 200 \mu \mathrm{V}$ was amplified $\left(0.5-2 \times 10^{3}\right)$, filtered $(50-3000 \mathrm{~Hz})$, and summed using an integrator (Neurolog; Digitimer Ltd., Welwyn Garden City, UK) with a time constant of $50 \mathrm{msec}$ to display the burst analysis. After recording the activity during spontaneous breathing, the animals were paralyzed with a neuromuscular blocking agent (Flaxedil; AAH Pharmaceuticals Ltd, Coventry, UK; $10 \mathrm{mg} / \mathrm{kg}$ gallamine triethiodide, i.v.) and artificially ventilated with a mixture of room air (50\%) and oxygen $(50 \%)$ at a rate of $40-60$ per minute (tidal volume, $2-4 \mathrm{ml}$ ). The purpose of this maneuver is to avoid any possible peripheral input (e.g., from the contralateral diaphragm) and thus ensure that any recorded phrenic rhythm is of central origin. To provoke the brainstem respiratory centers to their maximal output, recording was continued while the ventilator was temporarily stopped $(20-50 \mathrm{sec})$. This was to ensure that the conditions of our experiment did not elicit rhythmic supraspinal respiratory activity in latent crossed pathways in the lesion-alone animals, and therefore, that any activity observed in the phrenic nerve ipsilateral to the lesion was dependent on the presence of transplanted OECs.

Climbing. Two weeks after the operation, rats were tested by allowing them to climb twice up an inclined, 1-m-long grid at an angle of $15^{\circ}$ to the vertical. Climbing the grid is a spontaneous response acquired easily, and requiring neither compulsion nor reward. The climbing test was repeated three times a week for 7 successive weeks. Unoperated animals climb readily, with all four paws locating and grasping the bars without fault. In all operated animals, the forepaws and the hindpaws on the operated side showed varying degrees of difficulty in locating the grid bars. As a result, the forepaws overshot the bars to a depth varying from the wrist to the axilla. The rat sensed the overshoot, the paw was withdrawn, and the rat explored until it made contact with the bar, which was then grasped as normal and used as an anchor for the propulsive phase of the climb. In the disadvantaged paws, the propulsive force needed for climbing appeared normal, and the strength of the grasp (assessed by pulling the rats off the grid) was equal on the two sides. To distinguish between lesionalone and the lesion plus transplant groups of animals, the last of the three weekly tests was video-recorded for each of the 6 successive weeks from the third to the eighth week postoperatively. The faults in the forepaw on the operated side were scored by a $1 / 25$ th split-frame video analysis of all records, with a score of 1 for overshooting through the grid to the level of the wrist, 2 to the elbow, and 3 to the axilla. This method of scoring is objective, based on permanent records, and gives consistent values for different observers.

Histology. After the 6 weeks of serial functional testing for climbing, for terminal electrophysiology of the phrenic nerves, or both, the rats were perfused with $0.1 \mathrm{M}$ PBS, and the extent of the lesion in each animal was assessed by reconstruction from $20-\mu \mathrm{m}$-thick serial longitudinal cryostat sections taken through the entire width of the spinal cord at the level of the lesions/transplants and fixed on slides with acid-alcohol; alternate sections were stained with aqueous thionin or immunostained for neurofilament (Anti-Pan, 1:1000; Sternberger, Lutherville, MD) and counterstained with propidium iodide (Li et al., 1998).

\section{Results}

Hemisection produces consistent lesion with minimal damage to surrounding intact tissue, and the extent of the lesion in each individual was assessed by reconstruction from serial section histology. The hemisections, even those transecting the central canal and reaching beyond the midline, healed with minimal scarring, and no cyst formation or persistent macrophage invasion (Fig. $1 A, B)$. The matrix transplant method resulted in highly effective retention of the transplanted cells in the lesion site (Fig. 2). Comparison of Figure $2 A$ and $B$ shows no diminution or diffusion of the transplanted cell mass from 3 to $10 \mathrm{~d}$, by which time the cells show clear rostrocaudal alignment and migration into the host fiber tracts.

Immediately after the operation, all animals showed an unsteady gait and reduced responses to pinch of the contralateral hindpaw. In both lesion-alone and lesion plus transplant animals, all symptoms improved over the first week after operation. This improvement was markedly more rapid in the transplanted animals, but by 2 weeks, both groups had reached a level of cage behavior that was indistinguishable from each other and from that of unoperated rats. 

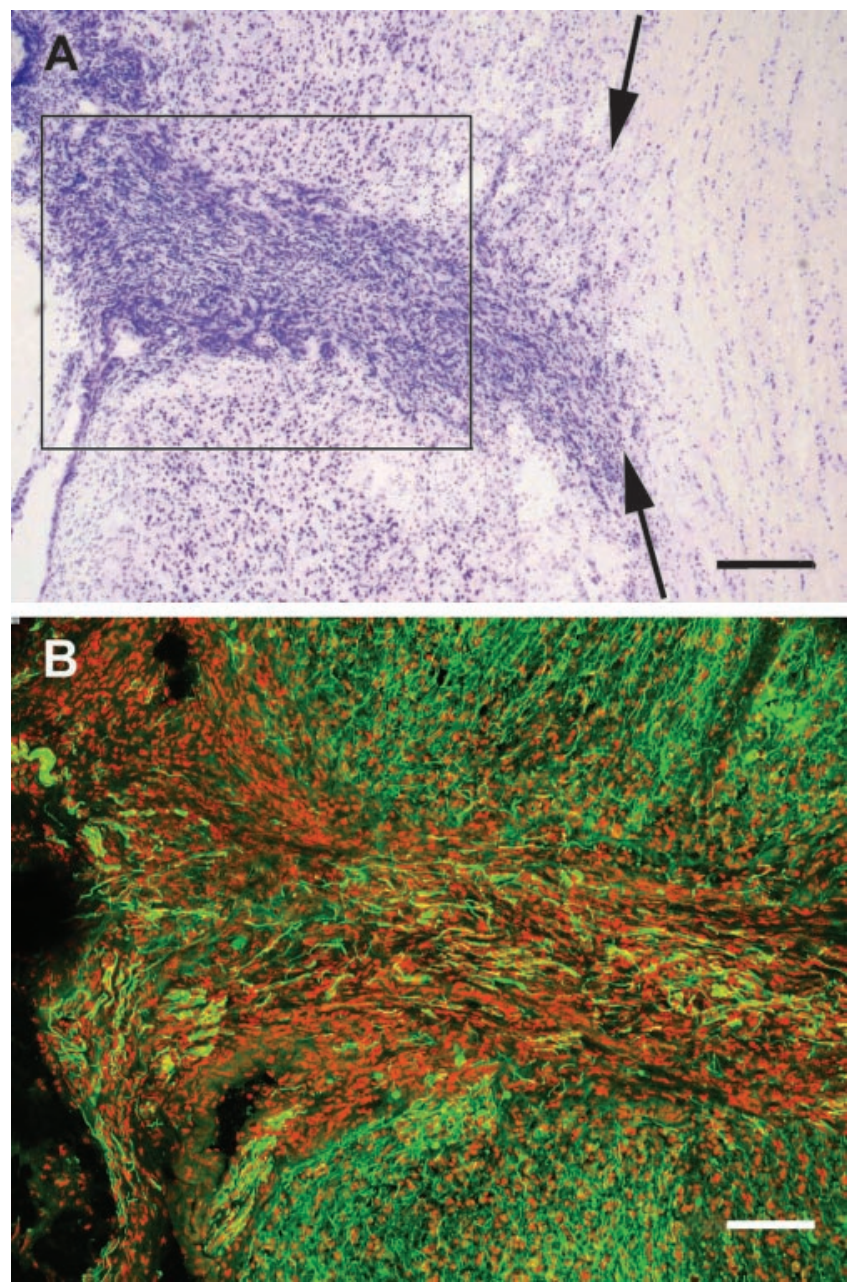

Figure 1. Horizontal sections through the mid-dorsoventral level of the spinal cord (top is rostral, left edge is the lateral edge of the spinal cord). A, Thionin; arrows indicate midline. $B$, Confocal image of the boxed area in $A$ from an adjacent section stained with neurofilament immunohistochemistry ( green) and counterstained with propidium iodide (red). Section thickness is $20 \mu \mathrm{m}$; survival time, 2 months. Scale bars: $A, 250 \mu \mathrm{m} ; B, 100 \mu \mathrm{m}$.

\section{Breathing}

A series of 23 hemisected animals was used to investigate the effects of lesions (Fig. 3) on the supraspinal control of the respiratory rhythm in the phrenic nerve after 2 months of survival. Serial section thionin and neurofilament histology showed that 14 of these animals had complete hemisections that destroyed the entire half of the spinal cord, reaching at least as far as the midline (Fig. 3B). In a terminal procedure under anesthesia, the electrical activity of the phrenic nerves was recorded (Fig. 3D). All 14 animals with complete hemisection had total unilateral loss of the supraspinal respiratory rhythm in the phrenic nerve on the operated side both during spontaneous breathing and also on a ventilator after curarization (to block any peripheral input) and brief asphyxial stress (to maximize the discharges in the descending supraspinal pathways). In nine animals in which the histological analysis showed that the hemisections were incomplete, the lesions had severed the lateral and dorsal parts of the cord but had spared the ventral funiculus (Fig. 3A). In all of these cases, the respiratory rhythm was present in the phrenic nerve of the operated side. This is in agreement with published evidence for a ventrolateral location of the descending bulbospinal respiratory pathways (Feldman et al., 1985; Ellenberger and Feldman, 1988; Castro-Moure and Goshgarian, 1996).
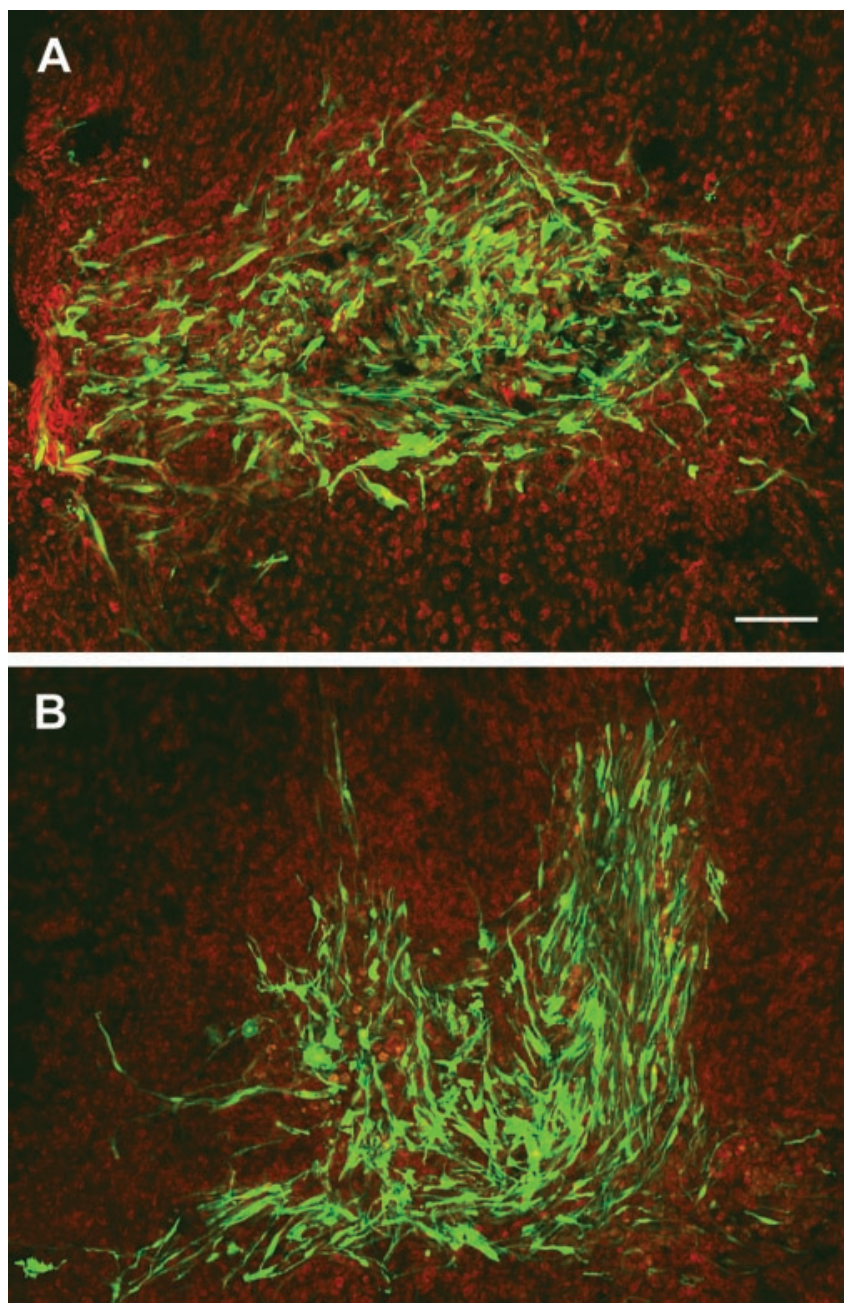

Figure 2. Transplanted OECs labeled with an adenoviral GFP construct form a large and dense meshwork in the lesioned area at $3 \mathrm{~d}(A)$ and $10 \mathrm{~d}(B)$ after operation, by which time the cells have adopted an elongated shape, aligned rostrocaudally. This shows that the cells are efficiently retained and remain clustered in the transplant site. Confocal image, green fluorescence, $0 \mathrm{ECs}$; counterstain propidium iodide, red. Section thickness, $100 \mu \mathrm{m}$. Scale bar, $100 \mu \mathrm{m}$.

In 24 animals, cells were transplanted into the hemisections. Subsequent histology confirmed that all of these hemisections completely destroyed the ventral funiculus. [The absence of incomplete lesions in the group of lesion plus transplant animals is the result of the additional damage caused by inserting the transplants (Fig. 3C, also see Fig. $4 C$ for the same effect).] On terminal examination after 2 months of survival, 19 of these lesion plus transplant animals showed a clear respiratory rhythm in the phrenic nerve during spontaneous breathing, and in 17 of these, the rhythm was maintained during curarization and asphyxia (Fig. 3D).

\section{Climbing}

During the period from 10 to $20 \mathrm{~d}$ after surgery, an improvement in climbing skill was observed, with the animals in the transplanted group clearly recovering more rapidly than those with lesions alone. From 3 weeks onward, individual scores for each animal in both groups showed a stable weekly fault score with neither improvement nor deterioration over the 6 weeks of the tested period. For 14 lesion-alone rats in which the subsequent histology indicated that the lesion had completely destroyed the dorsal columns (Fig. $4 B, D$ ), the average fault score for the six 


\section{BREATHING}

Lesions alone

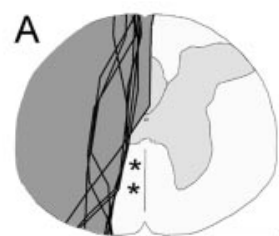

Breathing

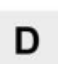

D

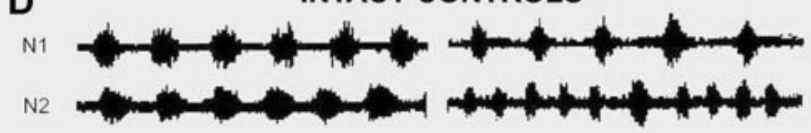

LESIONS ALONE

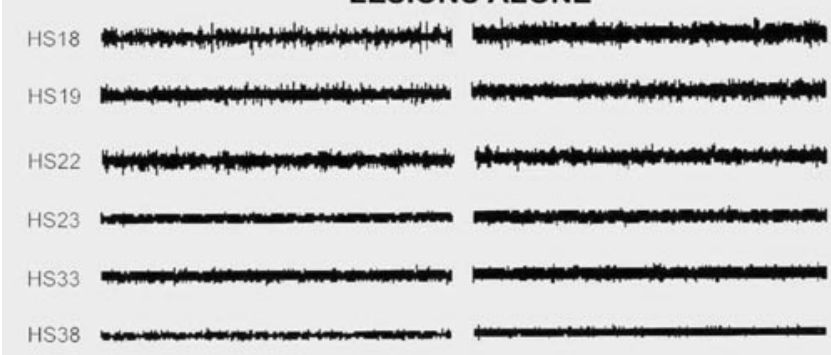

LESIONS WITH TRANSPLANTS

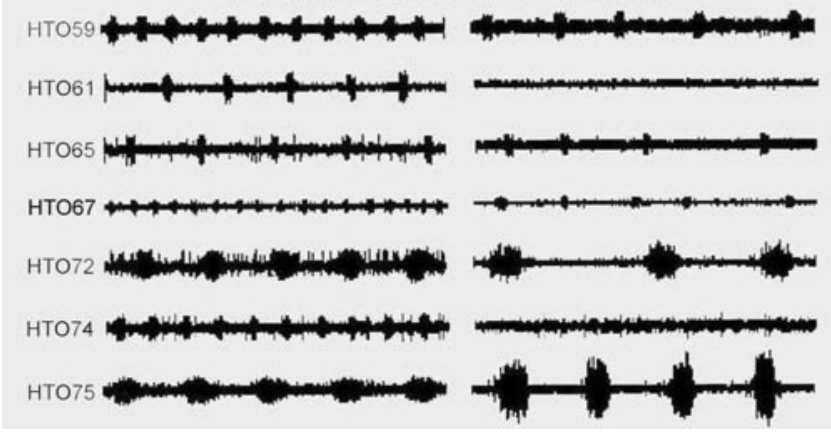

Figure 3. The extent of the hemisections is plotted in gray on the left, with the medial boundary of the lesions marked. Each line represents a different animal. $A$, Lesions that spare the ventral white columns ${ }^{* *}$ ) also spare respiratory rhythm in the ipsilateral phrenic nerve. $B$, Lesions that abolish the rhythm. $C$, Transplanted lesioned rats in which the hemisections are equal to or larger than those in $B$, but the rhythm is present because of the presence of the transplants. Scale bar, $1 \mathrm{~mm}$. D, Electrophysiological recording of the rhythmic compound action potential from the phrenic nerve in unoperated animals ("intact controls" shows two representative cases). The rhythm is abolished in animals with complete hemisections that include the ipsilateral ventral funiculus $(B)$; "lesions alone” shows a representative 5 of this group of 14 . The rhythm is present in 19 animals (C shows 5 representative cases) that have equally complete or even larger lesions but that also received transplanted 0ECs ("lesions with transplants"). Left column shows recordings made during spontaneous breathing. Right column shows recordings after curarization and $20-50$ sec of asphyxia.

weekly totals was $226 \pm 18$. In five lesion-alone animals in which the subsequent histology showed that lesion had spared a major part of the dorsal columns (Fig. 3A), the climbing deficit was less severe (group mean, $79 \pm 15$; Student's $t$ test $=6.3$; $p<0.0001$ ).

The group of 23 lesion plus transplant animals all had lesions that completely destroyed the dorsal columns. In the climbing test, the 23 lesion plus transplant group animals had a mean score of $55 \pm 7$ (Fig. $4 C, E$; Student's $t$ test for comparison with lesionalone including dorsal columns $=8.89 ; p<0.0001$ ). Of these, apart from one exception, the remaining 22 rats all had consid-

\section{CLIMBING}
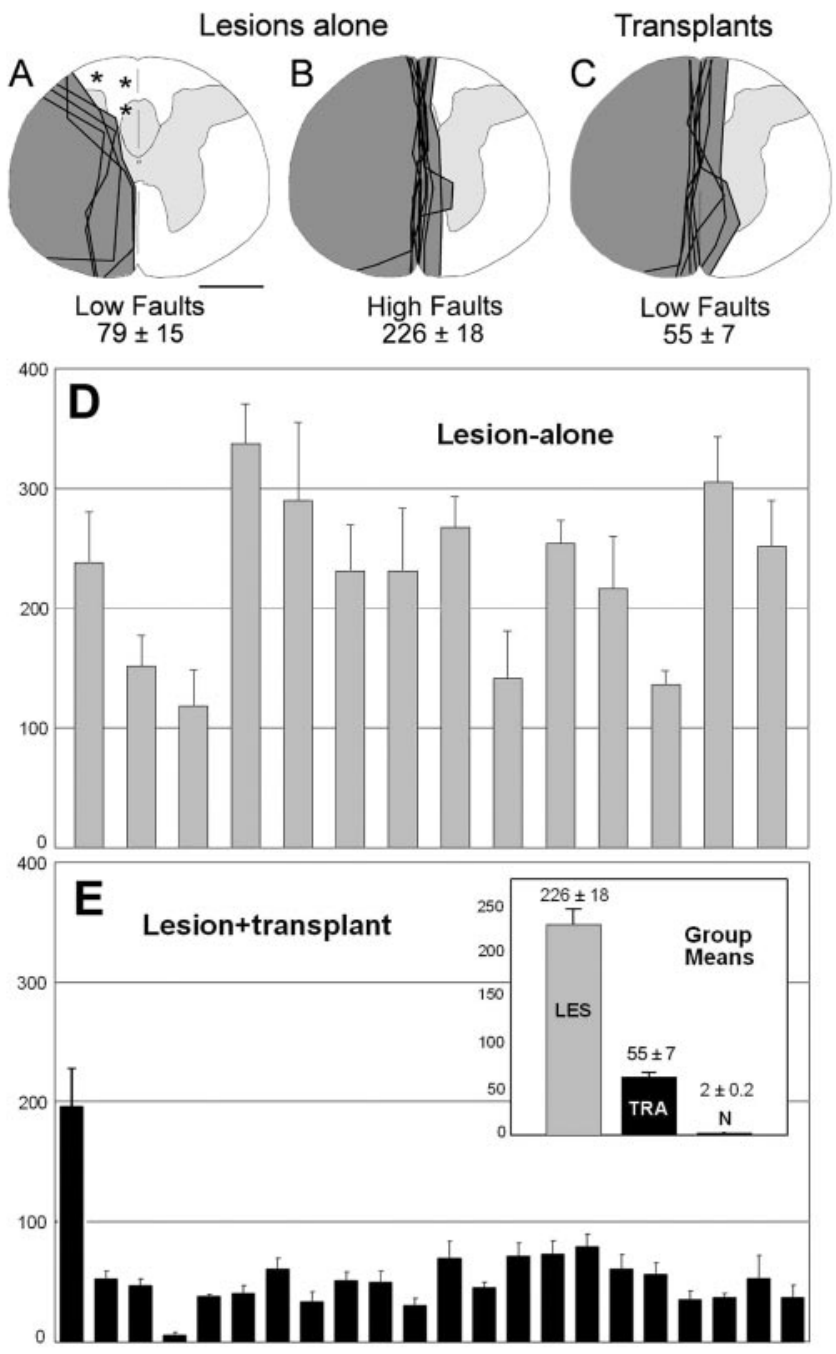

Figure 4. The extent of the hemisections, represented as in Figure 3. $A$, Lesions that give a lower climbing fault score $(79 \pm 15)$ and spare the region $\left(^{* * *}\right)$ of the dorsal columns and corticospinal tract. $B$, Lesions that are complete hemisections and give the highest fault scores (226 \pm 18). C, Transplanted lesions with complete hemisections in which the fault score (55 \pm 7) is reduced by the presence of the transplants. Scale bar, $1 \mathrm{~mm}$. D, E, Faults in the use of the ipsilateral forepaw for climbing. The total fault score for two measured test climbs (average of 6 weekly tests) for the 14 individual animals in the lesion-alone group ( $D$, gray bars) and the 23 individual animals in the lesion plus transplant group ( $E$, black bars) is shown; inset compares group means \pm SEM for lesion-alone (LES, gray), lesion plus transplant (TRA, black), and normal, unoperated $(N)$.

erably lower fault scores than the lesion-alone group. None of the transplanted animals reached the performance level of unoperated animals $(2 \pm 0.2 ; n=6)$.

\section{Discussion}

\section{General condition}

In evaluating functional outcomes in general, it is important to realize that rat spinal injuries do not produce the same degree of incapacity as human injuries, and also that in both rats and humans, major functional improvements occur spontaneously, and can be greatly enhanced by rehabilitative measures, without any reparative interventions in the spinal cord. The transplantation of cells is by no means the only cause of recovery in lesioned animals. In their general cage mobility, all lesioned animals with or without transplants improved greatly during the first $10 \mathrm{~d}$ after 
operation. Possible factors contributing to this improvement include reduction of edema, clearing of debris, vascularization, sprouting of new connections in denervated areas, and reprogramming of existing connections. Our observations show that after the initial period, however, the lesioned animals have persistent deficits in important complex functions, such as breathing and climbing.

\section{Breathing}

Nantwi and Goshgarian (2001) have extensively studied the "crossed phrenic phenomenon" in which activation of a latent, crossed-descending supraspinal respiratory pathway by section of the contralateral phrenic nerve, or by administration of alkylxanthines, restores function in the phrenic nerve and hemidiaphragm ipsilateral to a high cervical hemisection. Under the curarization and brief asphyxial conditions of our experiment, no activity appeared in the ipsilateral phrenic nerve in any of the 14 rats with complete hemisections involving the ventral funiculus. The return of supraspinal respiratory rhythm in our 19 transplanted animals with complete hemisections is therefore absolutely dependent on the presence of the transplanted OECs. Whether this recovery is caused by regeneration of severed ipsilateral supraspinal connections across the $\mathrm{C} 2$ lesion or by activation or sprouting of crossed pathways (Goshgarian et al., 1991) remains for further study. But whichever the mechanism may be, a significant difference is that whereas the crossed phrenic phenomenon is elicited only under conditions of respiratory stress, the transplants restore the ipsilateral rhythm (O'Hara and Goshgarian, 1991) during spontaneous breathing under unstressed conditions, requiring no drug administration, and the repair is presumably maintained and permanent.

\section{Climbing}

An overall improvement in hindlimb function during climbing has been reported in animals with OECs transplanted into complete midthoracic spinal cord transections (Ramón-Cueto et al., 2000). These animals, however, are severely disabled, and require intensive postoperative maintenance. The present study indicates that more precisely quantifiable climbing deficits can be consistently produced and studied in the ipsilateral forepaw after upper cervical hemisections, which leave the animals in overall much better condition, and demonstrate reliable and robust improvement after transplantation. This provides a simpler experimental model for studying the effects of different candidate reparative cell types and molecular interventions (Thallmair et al., 1998; Tang et al., 2001; Bradbury et al., 2002).

The histological analysis of the incomplete lesions indicates that the return of breathing reflects repair of damage to ventrally located tracts, and the return of climbing reflects repair of damage to dorsally located fiber tracts. These beneficial effects of the transplanted OECs could be attributable either to regeneration of cut fibers (as reported in other studies (Li et al., 1997, 1998) or to sprouting of uncut fibers (Thallmair et al., 1998), in either case restoring access to neural information otherwise made unavailable by the lesion. The demonstration that transplantation can simultaneously repair two quite independent spinal functions is encouraging for the future use of autografts in human spinal cord injuries.

\section{References}

Bradbury EJ, Moon LDF, Popat RJ, King VR, Bennett GS, Patel PN, Fawcett JW, McMahon SB (2002) Chondroitinase ABC promotes functional recovery after spinal cord injury. Nature 416:636-640.

Castro-Moure F, Goshgarian HG (1996) Reversible cervical hemispinalization of the rat spinal cord by a cooling device. Exp Neurol 141:102-112.

Decherchi P, Lammari-Barreault N, Gauthier P (1996) Regeneration of respiratory pathways within spinal peripheral nerve grafts. Exp Neurol 137:1-14.

Ellenberger HH, Feldman JL (1988) Monosynaptic transmission of respiratory drive to phrenic motoneurons from brainstem bulbospinal neurons in rats. J Comp Neurol 269:47-57.

Feldman JL, Loewy AD, Speck DF (1985) Projections from the ventral respiratory group to phrenic and intercostal motoneurons in cat: an autoradiographic study. J Neurosci 5:1993-2000.

Goshgarian HG, Ellenberger HH, Feldman JL (1991) Decussation of bulbospinal respiratory axons at the level of the phrenic nuclei in adult rats: a possible substrate for the crossed phrenic phenomenon. Exp Neurol 111:135-139.

Li Y, Field PM, Raisman G (1997) Repair of adult rat corticospinal tract by transplants of olfactory ensheathing cells. Science 277:2000-2002.

Li Y, Field PM, Raisman G (1998) Regeneration of adult rat corticospinal axons induced by transplanted olfactory ensheathing cells. J Neurosci 18:10514-10524.

Lu J, Féron F, Mackay-Sim A, Waite PME (2002) Olfactory ensheathing cells promote locomotor recovery after delayed transplantation into transected spinal cord. Brain 125:14-21.

Nantwi KD, Goshgarian HG (2001) Alkylxanthine-induced recovery of respiratory function following cervical spinal cord injury in adult rats. Exp Neurol 168:123-134.

O'Hara TEJ, Goshgarian HG (1991) Quantitative assessment of phrenic nerve functional recovery mediated by the crossed phrenic reflex at various time intervals after spinal cord injury. Exp Neurol 111:244-250.

Raisman G (2001) Olfactory ensheathing cells_-another miracle cure for spinal cord injury? Nat Rev Neurosci 2:369-375.

Ramón-Cueto A, Cordero MI, Santos-Benito FF, Avila J (2000) Functional recovery of paraplegic rats and motor axon regeneration in their spinal cords by olfactory ensheathing glia. Neuron 25:425-435.

Ruitenberg MJ, Plant GW, Christensen CL, Blits B, Niclou SP, Harvey AR, Boer GJ, Verhaagen J (2002) Viral vector-mediated gene expression in olfactory ensheathing glia implants in the lesioned rat spinal cord. Gene Ther 9:135-146.

Tang S, Qiu J, Nikulina E, Filbin MT (2001) Soluble myelin-associated glycoprotein released from damaged white matter inhibits axonal regeneration. Mol Cell Neurosci 18:259-269.

Thallmair M, Metz GA, Z'Graggen WJ, Raineteau O, Kartje GL, Schwab ME (1998) Neurite growth inhibitors restrict plasticity and functional recovery following corticospinal tract lesions. Nat Neurosci 1:124-131. 